\title{
La Société d'encouragement pour l'industrie nationale et le Conservatoire des arts et métiers (1801-1811)
}

Promoting Innovation beside the Conservatoire des Arts et Métiers: the early years of the Société d'Encouragement pour l'Industrie Nationale (1801-1811)

\section{Daniel Blouin et Gérard Emptoz}

\section{OpenEdition Journals}

Édition électronique

URL : http://journals.openedition.org/artefact/3933

DOI : 10.4000/artefact.3933

ISSN : 2606-9245

Éditeur :

Association Artefact. Techniques histoire et sciences humaines, Presses universitaires du Midi

Édition imprimée

Date de publication : 15 octobre 2019

Pagination : 75-95

ISBN : 978-2-8107-0650-1

ISSN : 2273-0753

\section{Référence électronique}

Daniel Blouin et Gérard Emptoz, « La Société d'encouragement pour l'industrie nationale et le Conservatoire des arts et métiers (1801-1811) », Artefact [En ligne], 10 | 2019, mis en ligne le 06 août 2020, consulté le 27 novembre 2020. URL : http://journals.openedition.org/artefact/3933 ; DOI : https://doi.org/10.4000/artefact.3933

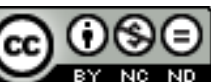

Artefact, Techniques, histoire et sciences humaines est mise à disposition selon les termes de la Licence Creative Commons Attribution - Pas d'Utilisation Commerciale - Pas de Modification 4.0 International 


\section{La Société d'encouragement pour l'industrie nationale et le Conservatoire des arts et métiers (1801-1811)}

\section{Daniel Blouin et Gérard Emptoz}

\section{Résumé}

L'article interroge la proximité, longtemps soulignée par les historiens, entre la Société d'encouragement pour l'industrie nationale et le Conservatoire des arts et métiers dans la première décennie du xix siècle, en s'appuyant sur un approfondissement de nos connaissances. Il souligne le rôle de son premier président, Chaptal, dans la conception d'une institution originale, d'abord par son utilitarisme et son souci de communication, ensuite par l'ampleur de ses champs d'action, qui explique le recours à des experts, notamment ceux du Conservatoire, aux compétences multiples.

\section{Mots-clés}

Conservatoire des arts et métiers, diffusion des savoirs, invention technique, Jean-Antoine Chaptal (1756-1832), Société d'encouragement pour l'industrie nationale

99 Daniel Blouin et Gérard Emptoz, « La Société d'encouragement pour l'industrie nationale et le Conservatoire des arts et métiers (1801-1811) », Artefact, 10, 2019, p. 75-95. 


\section{Promoting Innovation beside the Conservatoire des Arts et Métiers: the early years of the Société d'Encouragement pour l'Industrie Nationale (1801-1811)}

\section{Abstract}

Drawing from a deepening of our knowledge, the article questions the closeness, a long time underlined by historians, between the Société d'Encouragement pour l'Industrie Nationale and the Conservatoire des Arts et Métiers in the first decade of the nineteenth century. It highlights the role of its first chairman, Chaptal, in the design of an institution remarkable first by its utilitarism and focus on communication; remarkable also by the breadth of its endeavours, relying on the multiple competences of its experts, including specifically the Conservatoire's.

\section{Keywords}

Conservatoire des arts et métiers (Paris), Jean-Antoine Chaptal (1756-1832), diffusion of knowledge, Société d'encouragement pour l'industrie nationale (Paris), technical invention 


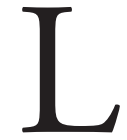

a Société d'encouragement pour l'industrie nationale et le Conservatoire des arts et métiers sont des institutions a priori dissemblables : l'une, association privée, l'autre, organisme d'État ; des dates de fondation, des contextes et des initiateurs différents. Pourtant, elles peuvent être rapprochées : intervenant toutes les deux dans le domaine des techniques, elles se vouent à l'encouragement, la distinction de l'invention utile et sa valorisation pour la socialiser, contribuer à en faire de l'innovation. Cette proximité a pu être soulignée, en son temps, par René Tresse, qui, en partant principalement des archives du Conservatoire, a montré le rôle de ses dirigeants, notamment celui de son administrateur, Claude Pierre Molard, dans la création de la Société d'encouragement et dans le développement de ses activités, durant la période du Consulat et du Premier Empire ${ }^{1}$.

À l'occasion du colloque consacré aux « jeunes années du CNAM », notre intention est de montrer ce que la Société naissante a de spécifique, à la fois dans sa conception, ses modes d'action et son évolution. Nous pouvons nous appuyer, pour ce faire, sur l'approche renouvelée des historiens, en ce qui concerne les institutions vouées à l'encouragement, sociétés savantes ou organismes officiels, leurs objectifs et de leur typologie, depuis leur essor au $\mathrm{XVIII}^{\mathrm{e}}$ siècle $^{2}$; nous disposons également d'études relativement récentes sur les premiers temps de la Société d'encouragement, basées toutefois uniquement sur les sources du Conservatoire, ou sur des sources imprimées, notamment le bulletin de la Société ${ }^{3}$; les archives de la Société, qui étaient encore inaccessibles, il y a peu, ont été sauvegardées et inventoriées dans leur intégralité par nos soins ${ }^{4}$; nous pouvons désormais exploiter les dépouillements des registres de procès-verbaux des séances de son instance de décision, le conseil d'administration, ainsi que ceux des registres de comptes qui enregistraient les dépenses liées à l'encouragement. Pour cet article, en plus de dossiers ponctuels, nous avons également utilisé les dossiers, notamment annuels, du comité de mécaniques.

\footnotetext{
1. Tresse, 1952.

2. Hilaire-Perez, 2007 ; Smeaton, 1955 ; ainsi que De Place, 1988.

3. Redondi, 1988 ; Chassagne, 1989 ; ainsi que Butrica, 1998.

4. Commission..., 2011.

5. Archives de la Société d'encouragement (ASEIN) : GEN/R1/R2/R3/R7, procès-verbaux du Conseil, 1801-1810 ; FIN/R1, comptes, 1802-1825 ; CME/1/1-1/19, dossiers annuels du comité de mécanique, 1801-1820.
} 
Notre projet est d'abord de mettre en évidence ce que ces sources croisées peuvent nous apporter sur le caractère original de la Société, en examinant l'orientation que ses fondateurs entendent lui donner, notamment la question de la relation avec l'État, marquée par la figure de son président-ministre, Chaptal ; ensuite d'apporter une réponse plus précise à la question posée par René Tresse, reprise par d'autres historiens, de sa proximité avec le Conservatoire. Nous parcourrons les "jeunes années " de la Société, essentiellement sa première décennie, où se mettent en place son sociétariat, son organisation, ses modes d'action, alors que la mission qu'elle s'est assignée est mise à l'épreuve d'un environnement économique changeant, de la relance postrévolutionnaire à l'économie de guerre d'un Empire qui se veut continental.

\section{La Société d'encouragement : une institution originale par ses enjeux et par ses formes d'action}

\section{Un pôle de sociabilité prestigieux, un corps intermédiaire}

La Société d'encouragement pour l'industrie nationale est créée officiellement le 9 brumaire de l'an X (31 octobre 1801) ; son premier président est le savant chimiste, industriel et ministre de l'Intérieur du Consulat, Jean-Antoine Chaptal ; les quelque 600 personnes portées sur les listes d'adhérents dans un premier temps, près de 1000 à la fin de la décennie, qui se rassemblent autour de lui, viennent d'horizons divers ${ }^{6}$ : elle apparaît comme un pôle de sociabilité réunissant de grands notables éclairés dans la filiation des sociétés savantes du XVIII ${ }^{\mathrm{e}}$ siècle (Fig. 1), loin de l'institution étatique qu'est le Conservatoire. Elle apparaît également comme un de ces corps intermédiaires qu'affectionne le régime consulaire, point de contact entre ces grands notables et les détenteurs du pouvoir : les " principaux magistrats de la République ", Bonaparte en premier, sont parmi ses premiers adhérents, rejoints par de nombreux dignitaires, hauts fonctionnaires

6. ASEIN : GEN/1/19 à 1/21, listes des membres de la Société, 1801-1806 ; Chassagne, 1989, p. $151-162$. 
et haut gradés du régime consulaire ; leur présence lui confère un caractère prestigieux qu'elle conservera durablement.

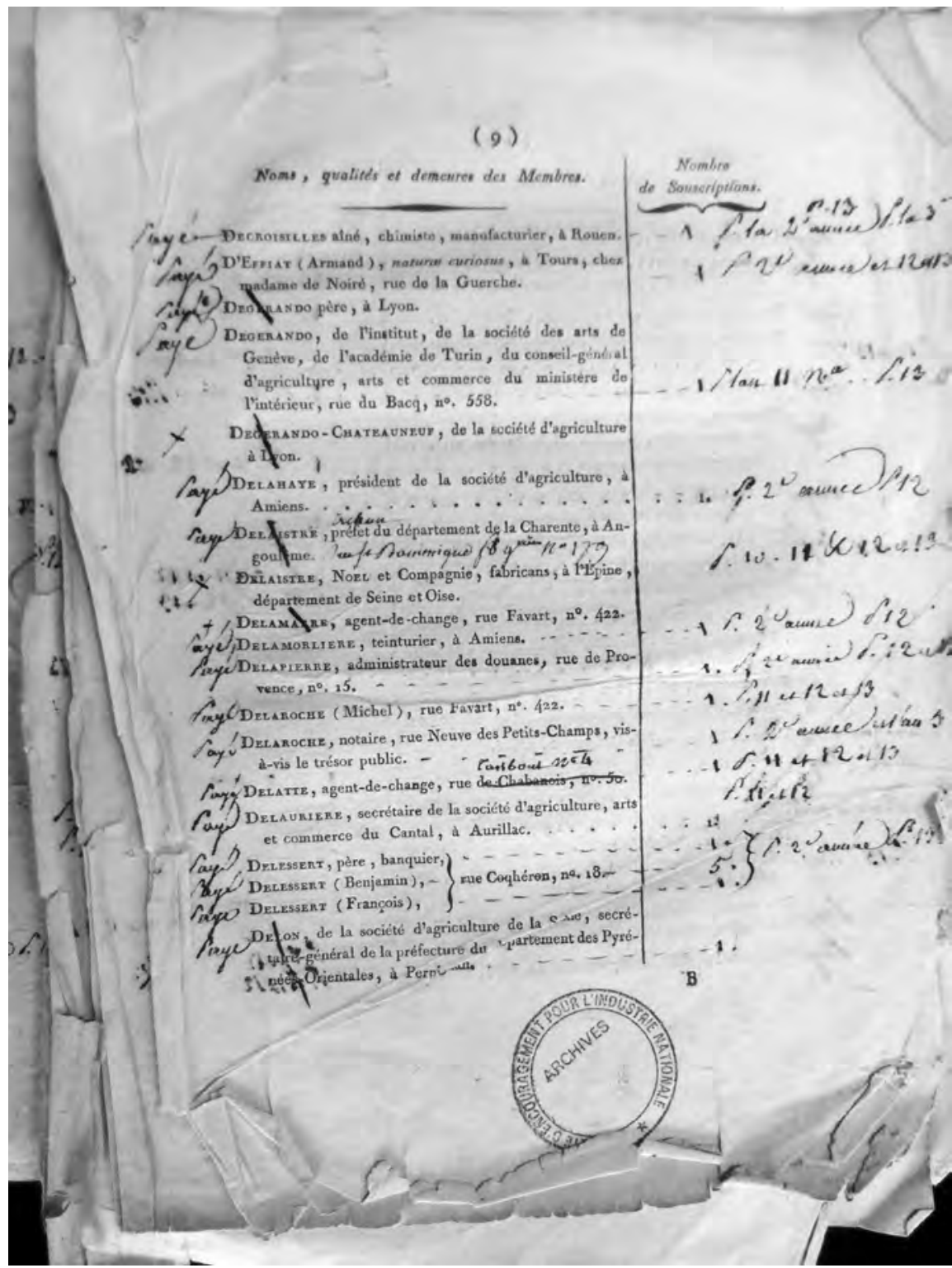

Fig. 1. - Extrait de la liste des membres composant la Société d'encouragement à la date du 9 nivôse an X (30 décembre 1801)

Archives de la Société d'encouragement, GEN-1/19. 


\section{Une Société née dans un « moment Chaptal »}

Les historiens n'ont pas manqué de souligner le caractère semi-officiel que Chaptal confère à la nouvelle institution : il l'installe au Louvre, dans la salle des délibérations du jury de l'exposition industrielle de l'an IX, qui s'est tenue peu de temps auparavant et qui y a laissé sa bibliothèque; il autorise en outre, d'emblée, la Société à utiliser la documentation technique du Conservatoire, et ultérieurement, celle détenue par son ministère ; enfin, lorsque la Société doit quitter le Louvre, il propose de l'installer dans les locaux de ce même Conservatoire, "afin de se donner des moyens mutuels d'appui ». La création de la Société semble donc inscrite dans un "moment Chaptal ", expression proposée récemment pour qualifier la période qui s'étend entre novembre 1800 et août $1804^{7}$ : elle est marquée par l'active politique de Chaptal, comme ministre de l'Intérieur, non seulement pour réorganiser les instances chargées de l'économie, mais aussi pour donner l'impulsion à une reprise de l'activité ; son enjeu principal est de combler le retard technique ressenti vis-à-vis de la Grande-Bretagne ${ }^{8}$. Au même titre que le soutien à l'installation et au développement du Conservatoire dans ses nouveaux locaux de Saint-Martin-des-Champs, la création de la Société, quelques semaines après l'exposition industrielle de l'an IX destinée à faire un bilan de l'industrie française, alors que les perspectives de paix peuvent contribuer à faciliter la socialisation des techniques de la puissance rivale, participe de ce projet.

\section{Un libéralisme de fond, un utilitarisme prééminent}

Comme le montrent les procès-verbaux, le débat au sein du conseil sur cette proposition débouche sur le refus de la majorité de ses membres de s'installer au Conservatoire, au motif que "cette installation n'offre point les convenances que l'on avait d'abord espérées "; plus qu'une question pratique, ce refus nous semble traduire une attitude de fond : la Société "libre ", ne saurait être confondue avec les institutions étatiques en matière d'encouragement. Cette vision est partagée par des proches de Chaptal : de Gérando, dans son discours de fondation de l'automne de l'an X, parle

7. Le Roux, 2011, p. 140-141.

8. Horn, 2006, p. 194-210. 
d'une société - à laquelle il accole, dans le projet rédigé quelques semaines auparavant, l'épithète de "libre »-créée dans "l'intérêt de l'industrie "; elle doit être, ajoute-t-il, " essentiellement distincte des travaux de l'administration ", car " un gouvernement éclairé [...] ne peut [...] tout voir ni tout faire $»$.

Chaptal se rallie à ce point de vue qui correspond à ses propres sentiments ${ }^{10}$; par ailleurs, fort de sa propre expérience d'entrepreneur, il souligne la " méfiance des ateliers ", envers « l'ignorance présomptueuse » des théoriciens : la nouvelle institution doit savoir être à l'écoute des premiers et éviter d'apparaître comme une académie : les mises à jour des listes de sociétaires montrent qu'au fur et à mesure que s'efface un groupe initial de financiers, attirés par une démarche spéculative, le groupe d'industriels appartenant à la frange la plus dynamique du patronat se renforce ; il compte déjà, à la fondation, des gens qui s'efforcent de constituer, par l'innovation et l'intégration, des empires industriels, comme les Périer, ou des industriels du luxe parisiens, comme l'ébéniste Jacob, dont le souci d'innover est tout aussi grand ; on voit s'ajouter rapidement de nouveaux noms, qui témoignent à la fois de la diversification des domaines que la Société peut aider, et de la demande de techniques nouvelles de la part des industriels, dans la chimie, dans la mécanique, dans la métallurgie, dans le domaine des arts du feu, enfin.

La première originalité de la Société réside dans cette dimension libérale et utilitariste : elle se réclame explicitement d'" une société fameuse en Angleterre ", la Society for the Encouragement of Arts, Manufactures and Commerce, fondée à Londres en $1754^{11}$.

\section{Un patronage étatique réel et paradoxalement nécessaire}

Tout en affirmant constamment son autonomie, la Société sera néanmoins largement et durablement subventionnée par le pouvoir : les comptes mettent en évidence l'importance des subventions fournies par le ministère de l'Intérieur à partir de 1804 ; elles représentent alors un cinquième du

9. ASEIN : GEN/1/1, Projet pour la Formation d'une Société.., 1801.

10. Chaptal, 1819, p. 214-215.

11. Hilaire-Pérez, 2007, p. 210-214. 
montant des souscriptions; elles permettent de développer les concours et surtout d'étoffer le bulletin. Les historiens avaient déjà noté que l'instance exécutive, le bureau, ainsi que le secrétariat, étaient peuplés de hauts fonctionnaires proches de Chaptal ; l'étude des délibérations du conseil souligne leur rôle dans l'animation de la nouvelle institution. Cette dernière doit épouser, par ailleurs, les options économiques du pouvoir, qu'elles soient en phase avec sa vision propre d'une industrie nationale génératrice de richesses, comme le développement de l'industrie textile, ou qu'elles soient dictées par la conjoncture politique, comme la recherche de produits de substitution dans le contexte du blocus; elle va travailler en étroite collaboration - on pourrait même dire imbrication, tant les deux institutions sont proches - avec le comité consultatif des arts et manufactures ${ }^{12}$. Elle se doit enfin de participer aux manifestations de valorisation organisées par l'État que sont les expositions industrielles ${ }^{13}$.

Il est difficile de se placer en dehors de l'activité d'un État qui se veut présent sur le front de l'économie : la Société peut néanmoins affirmer sa spécificité en apparaissant comme la meilleure représentante des attentes techniques concrètes des entrepreneurs. Enfin, pour lancer, puis pérenniser la nouvelle institution, dont les chances de survie paraissaient même à son fondateur, au premier abord, limitées ${ }^{14}$, l'aide matérielle de l'État comme la présence d'administrateurs compétents, sont nécessaires. Ce masque étatique assumé, qui cache un visage libéral, constitue la seconde originalité de la Société, notamment par rapport au Conservatoire, institution d'État.

\section{La brève option d'un local comme lieu de communication multiforme}

La Société, va louer à partir du printemps de l'an X, des locaux successifs au Faubourg-Saint-Germain, "aux marches du savoir et du pouvoir $»^{15}$ : elle veut les aménager en un centre actif de documentation et de médiation, doté d'un cabinet de lecture, vite dénommé bibliothèque ${ }^{16}$; il comprend également un cabinet de modèles et d'échantillons que l'on ambitionne

12. Demeulenaere-Douyère, 2016.

13. Butrica, 1998, p. 28-29.

14. Olivier, 1850, p. 10-11.

15. Blouin, 1996, p. 14-17.

16. Blouin et Emptoz, 2018, p. 25. 
même de transformer en " musée industriel "; expériences et démonstrations sont envisagées ${ }^{17}$. Le parallélisme avec le Conservatoire, lieu d'exposition et de démonstration, est ici évident. Mais cette ambitieuse politique axée sur le local, semble, d'après les procès-verbaux, passer au second plan, à partir de 1804, pour diverses raisons : le scepticisme affiché par Louis Costaz, très attaché à l'efficacité en relation avec l'objet social, envers le projet de conférences et de démonstrations, et qui redoute que la nouvelle institution "ne prenne un caractère trop marqué de discussion "; le manque de possibilités de rangement dans certains des locaux loués; les critiques de dirigeants, tel François-Augustin Silvestre, qui demandent le dépôt du cabinet de la Société au Conservatoire, pour constituer " une grande collection nationale »; enfin, le développement du bulletin. Elle se contentera de mettre en valeur, lors de ses séances générales bisannuelles, les objets primés, dans une démarche d'exposition officieuse, après la cessation des expositions officielles en 1806.

\section{Le choix rapide de la communication par le biais d'un bulletin bien documenté}

Les délibérations montrent qu'une publication est vite jugée par le conseil comme la meilleure façon de rendre compte de la globalité de l'évolution technique, et d'informer ses sociétaires de province : le bulletin de la Société d'encouragement pour l'industrie nationale est créé, à l'initiative du Genevois Pyrame de Candolle, en septembre 1802 ; il prend rapidement de l'ampleur, tant en nombre de pages que d'illustrations, et représente, dès 1804-1805, d'après les comptes, le quart des dépenses de la Société, valeur qui restera constante par la suite. Son format, ses gravures, l'abondance et la variété de son information, basée sur une documentation de qualité, notamment celle apportée par les périodiques étrangers, lui assurent une place qui sera longtemps sans équivalent dans le paysage de la diffusion de l'information technique française, voire internationale. Le Conservatoire ne possède pas, du fait de sa fonction première, un tel outil de diffusion, dont il ne se dotera que sous le général Morin, dans les années 1860. La fonction de veille technologique du bulletin est même renforcée, lorsqu'il publie, à partir de 1811, les dépôts de brevets français et de patents britanniques, alors que le Conservatoire, dont c'est pourtant

17. Sauvalle, 2017, p. 29-31. 
une des missions, ne publie qu'à partir de 1812 les descriptions des brevets expirés ou déchus. Ce soin apporté à la communication, notamment par la publication d'une information de qualité, nous paraît être la troisième manifestation de l'originalité de la Société.

\section{L'appui des ressources et des hommes du Conservatoire}

\section{Le souci d'une évaluation de qualité pour les inventions, dans le respect de la propriété industrielle}

La Société doit évaluer les inventions pour sélectionner celles qu'elle juge utiles. Tout en acceptant d'examiner parallèlement des inventions soumises par leurs auteurs, elle choisit d'organiser des concours, forme qui lui permet d'être seule juge des avancées qu'elle estime intéressantes, ainsi que des modalités d'évaluation (Fig. 2). Les programmes publiés annuellement et les comptes de la Société montrent toute la dynamique de cette forme d'action ; les récompenses décernées finissent par représenter $28 \%$ des dépenses de la Société. Il y a là une différence avec le Conservatoire, institution publique, qui ne prend en charge techniquement, comme en l'an XI ou en 1811, que des concours d'État, certes bien plus fortement dotés que ceux de la Société, mais portant sur des questions plus générales, la mécanisation de la filature du coton, puis de celle du lin ${ }^{18}$.

C'est aux experts qui forment le conseil de la Société, qu'incombe cette évaluation ; selon l'intention première de Chaptal, exprimée dans la séance générale de messidor an $\mathrm{X}$, elle doit prendre la forme d'un dialogue, sur un pied d'égalité, entre le "savant » et "l'artiste ", qui s'éclairent mutuellement ; cette conception, héritée des Lumières, disparaît toutefois très vite au profit d'une vision verticale, formulée par Louis Costaz au milieu de l'an XII : l'encouragement consiste à éclairer le producteur "des lumières de la science et de la technique ${ }^{19}$. Ce changement d'optique, en relation avec l'effacement du rôle de l'hôtel comme lieu d'information et de

18. Mercier, 2018, p. 264-265 et 361.

19. Costaz, Bulletin de la Société d'encouragement (BSEIN), 1804-1805, h.t., p. 2. 


\section{P R O R A M M I S \\ D $x$ s P $R I X$ proposés par la Société.}

Le Conseil d'ádministration croit devoir rappeler ici som 4 mairement les cinq sujets des yuix proposés dans la séance générale du 9 Nivose dernier.

Le premier, consistañt en une médaille et une somme de mille francs, est proposé pour une fabrication de filets pour la péche àmailles fixées par méchaniques ou antrea moyens économiques propres d̀ les établir d̀ un prix moindre que celui qui est prayé pour la fabrication des filets ordinaires.

Le second, de méme valeur, est proposé pour celui qui aura trouvé l'art de fabriquer le blanc de plomb avec perfection, et qui satisfasse pleinemeht aux besoins des arts, á un prix égal à celui des blanes de plomb étrangers les 1lus parfaits.

'Le troisième, consistant en une médaille et une somme de six cent francs, pour celui qui aura trouvé le moyen d'obtenir constamment et au prix du commerce, le bler de Prusse, d'une beauté et d'une nuance égales à celle des qualités les plus recherchées dans le commerce.

Le quatrième concours est composé de deux prix, l'uxs de mille et l'autre de six cent francs, pour les cultivateurs qui auront, au printemps de cette année, répiqué des grains d'automne dans un plus grand espace de terrein.

Fig. 2. - Extrait du premier programme de prix de la Société d'encouragement, publié le 9 nivôse an X (30 décembre 1801)

Bulletin de la Société d'encouragement pour l'industrie nationale, $1^{\text {re }}$ année, an XI (1801-1802), $1^{\text {re }}$ éd., vendémiaire an XI, p. 5.

\section{Cliché SEIN}

formation et avec l'importance croissante du bulletin, donne la primauté aux experts : la qualité de leur travail, basé fréquemment sur des essais, parait être le gage du maintien de l'attractivité de la Société aux yeux des producteurs, comme à ceux d'inventeurs attachés à son jugement. Cette évaluation, rendue publique, se veut néanmoins respectueuse du droit à 
la propriété industrielle, héritage de la Révolution ; la Société encourage même le dépôt de brevets, dont elle explicite la législation ${ }^{20}$.

\section{Un travail dans le cadre de comités ou d'équipes transversales}

Dès la fondation, ces experts sont répartis, à l'intérieur du conseil d'administration, entre cinq comités opérationnels spécialisés : " arts mécaniques ", " arts chimiques ", agriculture, "arts économiques ", qui traite des produits ou procédés de "l'économie domestique " destinés à l'équipement de la personne, de la maison ou de la ville, et enfin comité de " commerce ". Si, dans un premier temps, comme on l'a indiqué plus haut, experts d'État et scientifiques dominent, surtout dans les comités de mécanique et de chimie, Chaptal, qui entend assurer une large ouverture au monde de l'entreprise, s'efforce par la suite d'associer plus étroitement les manufacturiers à ces comités.

Dans le cas de la mécanique, à côté de l'ingénieur du génie Louis Costaz, de l'ingénieur des mines Baillet, d'un expert reconnu de l'industrie, François Bardel, on trouve d'emblée l'administrateur du Conservatoire Claude Pierre Molard et le démonstrateur Nicolas-Jacques Conté ; ils sont rejoints dès l'été 1802 par trois grands noms de l'industrie : Guillaume Ternaux pour le textile lainier, Jacques Augustin Périer pour les forges et la construction mécanique, Camille Pernon pour la soie ; quand Louis Costaz est appelé à des fonctions préfectorales en 1804, il est remplacé par l'horloger Breguet ; si l'on ajoute que, dans le premier cercle, Conté et Bardel sont également des industriels, on constate qu'outre la qualité, la professionnalisation est réelle dès les débuts de la décennie (Fig. 3).

Que ce soient pour les concours ou l'examen d'inventions, on constate, à la lecture des délibérations du conseil sur les programmes et des rapports, que ces experts travaillent souvent en équipes, ce qui garantit une certaine impartialité et autorise une plus grande largeur de vues. On remarque également le caractère transversal de nombreuses expertises, essentiellement sur les concours les plus importants, associant des membres de plusieurs comités pour juger à la fois de la qualité des matériaux, des procédés de fabrication et de la qualité du produit.

20. Costaz, BSEIN, 1802-1803. 


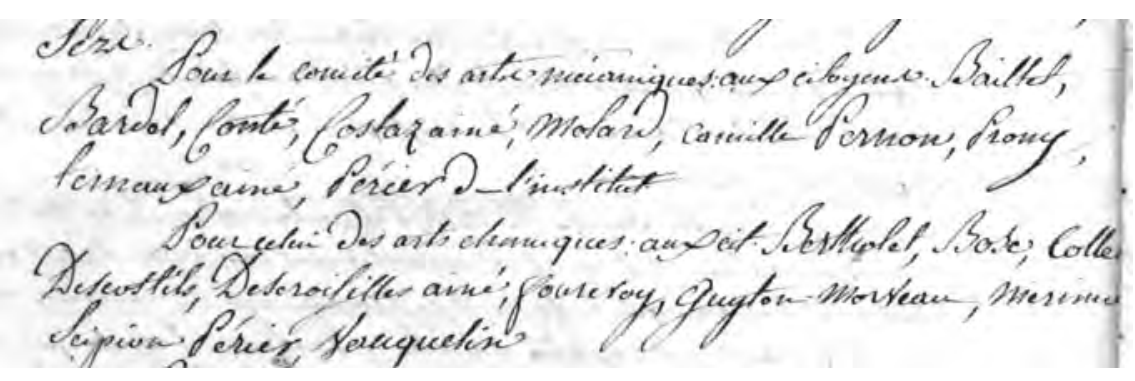

Fig. 3. - Composition des comités de mécanique et de chimie à la date du 9 messidor an X (28 juin 1802)

Archives de la Société d'encouragement, Procès-verbaux du Conseil d'administration, GEN-R1, séance du 9 messidor an X (28 juin 1802).

Cliché SEIN

\section{Des relations avec le Conservatoire pour la documentation, l'emploi des compétences et l'expérimentation}

Les relations personnelles entre les dirigeants des deux institutions ont été parfois tissées depuis longtemps, dans le cours de la Révolution, par le biais de la participation à des commissions techniques d'État, ou par des solidarités géographiques, dans le cas de Joseph Montgolfier. Ce dernier, que son ami de Gérando présentera comme l'un des initiateurs de la Sociétée ${ }^{21}$, est parmi ses premiers dirigeants, élu au comité des arts économiques.

Les ressources du Conservatoire, qui lui ont été ouvertes par Chaptal, ne sont pas négligeables pour la jeune Société : il lui offre une collection de machines et d'outillage sans équivalent, grâce aux efforts de Molard ; la Société y a recours, par exemple pour faire construire des modèles pour son cabinet, comme ceux de ces moulins à broyer et à hacher qu' elle désire envoyer en l'an XI à des sociétaires provinciaux, il détient en outre une documentation considérable, grâce notamment au bureau de dessinateurs ${ }^{22}$. Molard a également mis sur pied un atelier de mécanique se prêtant aisément aux essais : le Conservatoire est donc, au même titre que l'École polytechnique, la Monnaie, l'École des mines, l'École des ponts et chaussées, une des institutions qui accueille les expérimentations nécessaires.

21. De Gérando, BSEIN, 1814, p. 107.

22. Mercier, 2018, p. 235-353. 
Le cas de Jacquart illustre, quant à lui, une véritable coopération autour des talents d'un inventeur : la Société, qui organise en l'an X un concours sur une machine à fabriquer les filets, finance sa montée de Lyon à Paris, et le confie aux soins du Conservatoire, qui va l'employer dans l'atelier de mécanique; c'est là qu'il va construire, avec l'aide de Molard, une machine qui remporte ce premier concours l'année suivante, et qu'il va développer, pour un second concours lancé par la Société en l'an XIII et qu'il remporte à nouveau en 1808, la machine à fabriquer les étoffes brochées et façonnées, qui le rend célèbre ${ }^{23}$.

Cette collaboration s'étend à l'utilisation des locaux comme lieux d'expérimentation, selon la formule envisagée par la Société, comme l'illustre le cas du chauffage : sur un sujet qui passionne l'opinion éclairée depuis plusieurs décennies, au carrefour de la physique, pour ce qui est du rendement, de l'économie, pour ce qui est de la consommation de combustibles rares et aux usages disputés, et de la philanthropie, pour ce qui est de l'impact sur le bien-être des individus, des expériences scientifiques sur le rendement des systèmes de chauffage se tiennent successivement dans des locaux officiels, bureau de consultation des Arts, Conservatoire, et dans ceux de la Société, sous la direction de Montgolfier, entouré d'une commission officielle, mais composée de savants, d'ingénieurs et d'experts membres du conseil de la Société2 ${ }^{2}$. Cette collaboration se traduit, dans ce cas comme dans celui des résultats des concours d'état organisés par le Conservatoire, par la publication des résultats dans le bulletin de la Société. Mettant en commun des savoirs techniques, la Société et le Conservatoire mettent aussi en commun des experts qui se retrouvent dans une participation commune aux jurys des expositions industrielles de l'an X et de 1806, ils se manifestent surtout dans l'activité de la Société.

\section{La mécanique, Molard et la Société d'encouragement}

L'encouragement portant sur les machines, outils, procédés novateurs de la mécanique semble être le point de jonction naturel entre la Société et les dirigeants du Conservatoire : on a mentionné l'appartenance immédiate de

23. ASEIN : CME/20/97, dossier Jacquard, 1807-1808.

24. " Arts économiques... ", BSEIN, 1806-1807 et " Rapport... ", BSEIN, 1809. 
Molard et de Conté au comité de mécanique : le premier en est immédiatement désigné comme secrétaire, le second en sera plus tard le président, et, fort logiquement, ce comité choisit de se réunir au Conservatoire. Le lien est d'autant plus important que la mécanique apparaît rapidement, aux côtés de la chimie, comme une des activités phares de la Société, sans doute en raison de la nécessité du rattrapage sur l'Angleterre évoqué plus haut ; l'étude de l'importance relative des rapports, notes, articles publiés dans le bulletin et rattachés aux différents thèmes, montre sa part croissante : un tiers de l'espace rédactionnel dans la période de fondation, près de $40 \%$ aux débuts des années 1810, loin devant la chimie. Importante par sa couverture médiatique, la mécanique ne l'est pas moins par sa place dans les dépenses d'encouragement : avec $30 \%$, elle en constitue le premier poste sur l'ensemble de la période, devant les $20 \%$ de la chimie.

\section{PRIX PROPOSÉS POUR L'ANNEEE I809. \\ aRTS MÉCANIQUES.}

V].

Prix pour une petite machine à feu.

Le besoin des petites machines à feu est généralement senti. Leur usage est très limité, $\rho t$ si on en emploie plusieurs dont la force ne surpasse pas celle de quelques chevaux,

La Société d'encouragement, considérant l'utilité qui peut résulter de l'emploi des petites machines à feu, propose un prix de la valeur de six mille francs pour une petite machine à feu, dont la force équivaudra à celle capable d'élever à la hauteur d'un mètre un million de kilogrammes dans l'espace de douze heures, et dont la dépense totale, $y_{1}$ compris les intérêts du capital et les frais d'entretien, n'excédera pas, à Paris, la somme de 7 francs 5o centimes pendant ce même temps.

Ce prix sera décerné, dans la séance générale de janvier 1809 , da celui qui présentera une unachine en état de fonctionner, qui, à la meillenre construction, réunira les conditions ci-dessus énoncées.

Les machines devront être présentées au concours avant le $j^{\text {ex }}$ novembre 1808 .

Afin de faciliter les recherches de veox qui voudront concourir pour eet objet, la Société a cru devoir joindre ici une note de différens ouvrages à consulter, qui ont traité des pompes .et des machines mues par le feu.

Fig. 4. - Extrait du programme du concours des petites machines à feu rédigé par Molard et adopté dans la séance générale du 11 mars 1807

Bulletin de la Société d'encouragement pour l'industrie nationale, $5^{\mathrm{e}}$ année, 18061807, p. 238. 
Si l'activité de Conté, disparu en 1805, est limitée au sein du comité, Molard, par contre en reste un des acteurs incontournables durant 31 ans : "Ce que cet homme [...] a fait pour [la Société] est considérable, ce qu’il fait pour les arts mécaniques est immense " dit de Gérando dans son éloge en $1837^{25}$. Fréquemment associé avec Bardel en ce qui concerne les concours (Fig. 4), il est également très sollicité pour examiner des produits ou des procédés soumis à la Société : la liste en est longue et variée et témoigne de la diversité de ses pôles d'intérêt, souvent en relation avec la présence de modèles au Conservatoire, auxquels il se réfère. En plus de la publication de ses nombreux rapports, il rédige, entre 1802 et 1812, une cinquantaine d'articles de mécanique dans le bulletin, dépassé seulement en cela par Bardel.

Dans ses interventions devant le conseil ou dans ses publications, il imprime la marque de sa longue expérience personnelle, comme dans le cas des machines à filer la soie (Fig. 5) ; il lui arrive aussi de rappeler, à l'occasion de certains débats, l'existence de machines qu'il a conçues; dans les programmes qu'il rédige, il montre également l'étendue de son savoir technique, et la qualité de sa veille technologique personnelle, par exemple lorsqu'il fournit, pour le programme des petites machines à feu, en 1807, une bibliographie abondante et à jour ${ }^{26}$.

\section{En conclusion, une Société qui parvient vite à affirmer sa vision de l'innovation au service de l'industrie française}

Bien des aspects séparent le Conservatoire de la Société d'encouragement : d'un côté une institution d'État, dont la mission principale est la conservation, la présentation et la démonstration d'objets techniques, capable de dialoguer avec les producteurs de son proche environnement, la capitale, et qui commence également à entreprendre des activités de formation ; de l'autre une association, qui apparaît au premier chef comme un corps intermédiaire prestigieux tant par sa composition que par son patronage étatique, mais qui s'affirme vite comme un groupe, de plus en plus étoffé, d'experts ; se donnant comme espace d'activité, le territoire national,

25. De Gérando, BSEIN, 1837.

26. «Programme... », BSEIN, 1806-1807. 
comme domaine, l'ensemble des activités productives, elle entend non seulement examiner, mais également prendre l'initiative de la recherche et de la diffusion des techniques nouvelles.

\section{A R T S M E C H A N I Q U E S.}

DFS KLCIINZA FILZRIA BOI H.

Extralt d'un Rapport fait d la Sociellé d'Encouragemont, au nont du Comité des Arts méchäniques, par le C. Molard,

Le cocon dans lequel le ver a soie s'enveloppe, peut Atre considérí, velativement à la filature, comnno un poloton qu'il s'agit de dóvider. La réunion d'un certaia nombre de brins tirés d'autant de cocons, forne le fil de soie, Le nombre de brine varie suivant la grosseur que l'on veut Bonner au Al, L’égalité do la filature dépend principale. ment de l'attention, de la vigilance, et de l'adresse de la filause; mais il est d'autres qualités,non moing esşentielles, qui dépendent de la bopne construction des appareils employés au tirage des soies ; les appareils usités aujour. d'luui en France, se réduisent à trois; $1^{\circ}$. le tour ordi. paire; $a^{\circ}$. le tour de Vaucanson; $3^{\circ}$, le tour de Vaucanson arec les additions de Tabarin,

Les défauts inhórens \&े l'emploi du tour prdinaire, sont le mauvais croisage, le gommage et le vitrage,

Ira soie qui n'ent pas suffisanment croisée, manque de nerf, alle se casse au moindre effort qu'elle éprouve daps le devidage, c'ost un accident qui arfive souvent के la soie gommée, On dit yue la noie est vitrée lorsqu'elle est plitcée sur le tour do manière que tous les file sont couchés les uns sur les autres de maniere is se confondre; alors il est très difficile de sotrauyer at de disccerner le fil qui s'est rompu,

Vaucanson sorrigen ces trols défauts. Au moyen du double eroisage qu'il imagina, les brins, par leur frottement mutuel, sont intimément róunis en un aeul corps, Corment un fil plus nerveux, plus aprondi et presquesec,

Fig. 5. - Extrait du rapport de Molard sur les tours à soie devant le conseil, le 2 messidor an X (21 juin 1802), publié dans le premier numéro du bulletin

Bulletin de la Société d'encouragement pour l'industrie nationale, $1^{\mathrm{re}}$ année, an XI (1801-1802), $1^{\mathrm{re}}$ éd., vendémiaire an XI, p. 5. 
Reflet du libéralisme, du pragmatisme et du souci de communication du groupe des fondateurs, réunis autour de Chaptal, la nouvelle société s'appuie cependant sur l'État, ses moyens matériels et ses institutions techniques ; le Conservatoire est donc étroitement associé à sa création, par ses ressources, par les qualités d'analyse, l'expérience et l'activité de ses dirigeants, principalement dans le domaine de la mécanique. En retour, elle fournit, concurremment avec ce dernier, les experts des expositions industrielles organisées par l'État.

Lorsque Chaptal cesse d'exercer des responsabilités politiques, à partir de 1804, il conserve durablement une grande influence auprès des cercles dirigeants d'un État dont le rôle directeur reste essentiel dans l'économie nationale : il peut jouer de son prestige de savant et d'expert des questions industrielles, comme de sa vision d'une "industrie nationale " source de richesse. Il peut ainsi pérenniser sa création : pour lui donner un élan décisif, il utilise de manière optimale le capital humain dont elle dispose, pour des évaluations de qualité ; les hommes du Conservatoire sont alors de plus en plus impliqués dans des expertises transversales, qui peuvent mettre en jeu des travaux scientifiques, particulièrement pour la chimie ; il donne également un rôle accru au bulletin de la Société, conçu comme un outil global d'information technique, y compris pour les travaux faits au Conservatoire, de veille technologique, et même de bilan de l'industrie française. 
La Société d'encouragement pour l'industrie nationale...

\section{Bibliographie}

"Arts économiques. Expériences faites par ordre du gouvernement sur divers appareils de chauffage... ", Bulletin de la Société d'encouragement pour l'industrie nationale, vol. 5, 1806-1807, p. 108-119.

Blouin Daniel et Emptoz Gérard, "Constitution, usages et valorisation des collections de la Société d'encouragement pour l'industrie nationale ; un projet fondateur, une mise en œuvre tardive, une sauvegarde récente (années 18002000) ", dans Wenger Sylvain (dir.), Penser/Classer les collections des sociétés savantes, Slatkine, Genève, 2018, p. 19-30.

Blouin Daniel, "La Société d'encouragement pour l'industrie nationale, lieux et étapes I-Les premières implantations, les premières ambitions, les premières manifestations (vendémiaire an X/octobre 1801-juillet 1812) ", L'Industrie nationale, $1^{\text {er }}$ trimestre 1996, p. 11-21.

Butrica Andrew J., "Creating a Past: the Founding of the Société d'Encouragement pour l'Industrie Nationale, Yesterday and Today", The Public Historian, vol. 20, n 4, autumn 1998, p. 21-42.

Chaptal Jacques-Antoine, De l'industrie françoise, tome II, Renouard, Paris, 1819.

Commission d'histoire de la SEIN, Inventaire des archives de la Société d'encouragement pour l'industrie nationale, $2^{\mathrm{e}}$ éd. augmentée, La Société, 2011.

Costaz Louis, « Rapport fait à l'Assemblée générale, le 23 messidor an XII, [...] sur la nouvelle organisation du bulletin... ", Bulletin de la Société d'encouragement pour l'industrie nationale, vol. 3, 1804-1805, $2^{\mathrm{e}}$ éd., h.t. p. 1-4.

Costaz Claude-Anthelme, "Notice sur les brevets d'invention et sur la législation qui y est relative. ", Bulletin de la Société d'encouragement pour l'industrie nationale, vol. 1, 1802-1803, $2^{\mathrm{e}}$ éd., p. 81-85.

De GÉRAndo Joseph, " Notice sur M. Joseph Montgolfier... ", Bulletin de la Société d'encouragement pour l'industrie nationale, vol. 13, 1814, p. 91-108.

De Gérando Joseph, «Compte rendu des travaux de la Société d'encouragement depuis la séance générale du 6 juillet 1836 ", Bulletin de la Société d'encouragement pour l'industrie nationale, vol. 36, 1837, p. 298.

Demeulenaere-Douyère Christiane, "Le luxe sous l'Empire ou la question des matières premières indigènes ", dans CoQuery Natacha, Ebeling Jörg, Perrin-Khelissa Anne \& Sénéchal Philippe (dir.), Les progrès de l'industrie perfectionnée. Luxe, arts décoratifs et innovations de la Révolution française au Premier Empire, PUM, Toulouse, 2016, p. 37-43.

de Place Dominique, "Le Bureau de Consultation pour les Arts, Paris, 17911796 », History and Technology, 1988, vol. 5, p. 139-178. 
Hilaire-PÉrez Liliane, "Les sociabilités industrielles en France et en Angleterre au XVIIIe siècle : réseaux, institutions, enjeux ", dans Benoit Serge, EMPTOz Gérard et Woronoff Denis (dir.), Encourager l'innovation en France et en Europe Autour du bicentenaire de la Société d'encouragement pour l'industrie nationale, CTHS, Paris, 2007, p. 201-238.

Horn Jeff, The Path not Taken: French Industrialisation in the Age of Revolution, 1750-1830, The MIT Press, Cambridge (Mass.), 2006.

Le Roux Thomas, Le laboratoire des pollutions industrielles Paris 1770-1830, Albin Michel, Paris, 2011.

Mercier Alain, Le Conservatoire des Arts et Métiers des origines à la fin de la Restauration (1794-1830), Snoeck-Le CNAM, Paris, 2018.

Olivier Théodore, Histoire de la fondation de la Société d'encouragement pour l'industrie nationale..., Bouchard-Huzard, Paris, 1850.

"Programme de prix proposé par la Société d'encouragement dans sa séance générale du 11 mars 1807... Prix pour une petite machine à feu ", Bulletin de la Société d'encouragement pour l'industrie nationale, vol. 5, 1806-1807, p. 238-240.

"Rapport sur les expériences faites au Conservatoire des Arts et Métiers avec divers appareils de chauffage, d'après les ordres de S. Exc. le Ministre de l'intérieur, Bulletin de la Société d'encouragement pour l'industrie nationale, vol. 8, 1809, p. 23-27.

Redondi Pietro, "Nation et entreprise. La Société d'encouragement pour l'industrie nationale, 1801-1815 ", History and Technology, An International Journal, vol. 5, 1988, p. 193-222.

Sauvalle Chloé, "Les vicissitudes d'une collection d'objets techniques au XIX ${ }^{\mathrm{e}}$ siècle : le cabinet des machines de la Société d'encouragement pour l'industrie nationale ", Artefact, n' 5, 2017, p. 27-40.

Smeaton W. A., "The Early Years of the Lycée and the Lycée des Arts. A Chapter in the Lives of A.L. Lavoisier and A.F. de Fourcroy ", Annals of Science A Quaterly Review of the History of Science since the Renaissance, vol. 11, 3, nov. 1955 , p. 257-267 et vol. 11, 4, déc. 1955, p. 309-319.

Tresse René, "Le Conservatoire des Arts et Métiers et la Société d'Encouragement pour l'Industrie nationale au XIX ${ }^{\mathrm{e}}$ siècle ", Revue d'histoire des sciences et de leurs applications, 1952 , tome $\mathrm{V}, \mathrm{n}^{\circ} 3$, p. 246-263. 


\section{Les auteurs}

Daniel Blouin, professeur d'histoire-géographie en retraite, est l'un des fondateurs de la commission d'histoire de la Société d'encouragement, dont il a contribué à classer et à inventorier les archives. Il a par la suite effectué des recherches sur l'histoire de la Société et de ses locaux successifs, ainsi que sur certaines des techniques qu'elle a contribué à développer. Parmi ses publications : BLouin Daniel et Emptoz Gérard, « Constitution, usages et valorisation des collections de la Société d'encouragement pour l'industrie nationale ; un projet fondateur, une mise en œuvre tardive, une sauvegarde récente (années 1800-2000) », dans Wenger Sylvain (dir.), Penser/Classer les collections des sociétés savantes, Slatkine, Genève, 2018, p. 19-30.

Gérard Emptoz, docteur ès sciences d'État, professeur honoraire d'histoire des sciences et des techniques de l'université de Nantes, est l'auteur de publications sur l'histoire de l'innovation industrielle en chimie et sur l'histoire de la Société d'encouragement pour l'industrie nationale. Depuis 1996 il est membre de la Commission d'histoire de cette société, et il en est actuellement le président. Ses travaux récents sur cette histoire : Benoit Serge et EMPTOz Gérard, « Les collections de chimie de la Société d'encouragement », Revue d'Histoire des sciences, 69-1, 2016, p. 137-151 ; EмPToz Gérard, « Un groupe d'experts au service de la chimie et de ses industries : le Comité des arts chimiques de la Société d'encouragement pour l'industrie nationale », dans EMPTOz Gérard, FAUQue Danielle et BREYSSE Jacques (dir.), Entre reconstruction et mutations, les industries de la chimie entre les deux guerres, EDP Sciences, Paris, 2018, p. 137-164.

Contact commun : sein.histoire@gmail.com 